Abnormalities of epidermal growth factor receptor (EGFR) in non-small-cell lung cancer (NSCLC) patients consist of EGFR overexpression and EGFR (HER1) gene mutations. Structural dysfunction of the tyrosine kinase domain of EGFR is associated with the clinical response to tyrosine kinase inhibitors (TKI) in patients with NSCLC. The most common EGFR gene mutations occur as either deletions in exon 19 or as substitution L858R in exon 21 and cause a clinically beneficial response to gefinitib or erlotinib treatment. Unfortunately, the majority of patients finally develop resistance to these drugs. Acquired resistance is linked to secondary mutations localised in the EGFR gene, mainly substitution T790M in exon 20. Through intense research a few different mechanisms of resistance to reversible tyrosine kinase inhibitors have been identified: amplification of MET or IGF-1R genes, abnormalities of PTEN and mTOR proteins as well as rare mutations in EGFR and HER2 genes. Extensively investigated new drugs could be of significant efficiency in NSCLC patients with secondary resistance to reversible EGFR TKI.

Key words: epidermal growth factor receptor, EGFR gene mutations, tyrosine kinase inhibitors, resistance mechanisms.

\section{Mechanisms of resistance to reversible inhibitors of EGFR tyrosine kinase in non-small cell lung cancer}

\author{
Paweł Krawczyk ${ }^{1}$, Radosław Mlak ${ }^{1}$, Tomasz Powrózek ${ }^{1}$, Marcin Nicoś $^{1}$ \\ Dariusz M. Kowalski ${ }^{2}$, Kamila Wojas-Krawczyk ${ }^{1}$, Janusz Milanowski ${ }^{13}$
}

1Department of Pneumonology, Oncology and Allergology, Medical University of Lublin, Lublin, Poland

2Department of Lung Cancer and Thoracic, Oncology Centre - Maria Skłodowska-Curie Institute, Warsaw, Poland

${ }^{3}$ Institute of Agricultural Medicine, Lublin, Poland

\section{Introduction}

Lung cancer is the main cause of cancer deaths both in Poland and in the world [1]. Non-small cell lung cancer accounts for about $85 \%$ of all lung cancers, and the progress in its treatment is still not very satisfactory. The therapeutic procedure of choice is surgery, but only every fifth patient qualifies for it. This is due to the fact that most patients at the time of diagnosis are in an advanced stage of the disease, usually accompanied with tumour metastases. In about $25 \%$ of such patients extension of life by a few month is possible by using standard chemotherapy or chemo-and radiotherapy. Such disappointing results force us to search for new therapeutic options [2, 3].

One of the new molecular targets for non-small cell lung cancer (NSCLC) therapy is the epidermal growth factor receptor (EGFR). EGFR (HER1) belongs to the ErbB (HER) receptor family. For its activation, not only a connection with a suitable ligand is necessary, but also homo- or heterodimerisation on the cell surface with other receptors of the HER family (HER 2-4). The intracellular domain of EGFR has activity of tyrosine kinase and it is responsible for the phosphorylation of cellular proteins in the Pi3K/AKT signalling pathway. Furthermore, this domain has regulatory functions. In many cancers, including NSCLC, activating mutations or overexpression of the EGFR gene are found. In some cases progression of the disease - uncontrolled cell proliferation, inhibition of apoptosis, and the ability to metastasise - is the effect of excessive activation of intracellular pathways constantly stimulated by EGFR. Therefore, there is more and more interest in treatment associated with inhibition of EGFR [4-6].

\section{EGFR inhibitors in the treatment of non-small cell lung cancer}

In clinical practice, three mechanisms of inhibition of EGFR function are used. One of them is blocking of the extracellular domain of the receptor through monoclonal antibodies (cetuximab, panitumumab), which prevents connection of the EGFR ligand or receptor dimerisation. Blocking of signal transduction from the cell membrane to the nucleus by small molecule inhibitors of tyrosine and serine-threonine kinase is still in the experimental phase [9]. The biggest hopes for improving the prognosis in NSCLC are associated with the introduction of small molecule, reversible tyrosine kinase inhibitors of EGFR (EGFR TKI) gefitinib and erlotinib. The mechanism of their action is based on reversible binding to the intracellular tyrosine kinase domain of EGFR and blocking of ATP binding. Selective connectivity of the inhibitor prevents phosphorylation of the tyrosine kinase domain, and in consequence activates the pathway of 
cellular signal transduction, and leads to cell cycle arrest in G1 phase and increase in apoptosis of tumour cells. The therapeutic effect of EGFR TKI depends on the amino acid structure of the tyrosine kinase domain conditioned upon the state of the EGFR gene. Appearance of the most frequent activating mutation - deletion of 15 nucleotides in codons 746-750 in exon 19 and the L858R substitution in exon 21 of the EGFR gene - is associated with permanent stimulation of the receptor, but they also lead to an increase in both efficiency of reversible EGFR TKI and effectiveness of radiotherapy.

Activating mutations of the EGFR gene have been reported in only about $10 \%$ of Caucasians patients with NSCLC, more often in non-smokers, women, and patients with adenocarcinoma. Therefore, the first studies on the efficacy of erlotinib and gefitinib in second-line treatment (BR.21, ISEL, INTEREST) have shown that an objective response to EGFR TKI treatment occurs in less than $10 \%$ of patients in the general population [10-14].

In recent clinical trials (IPASS, OPTIMAL, EURTAC) conducted on carriers of activating mutations in the EGFR gene, over $70 \%$ response and almost one year progression-free survival (PFS) were reported. For this reason, both inhibitors were granted registration in the treatment of locally advanced and advanced NSCLC in first-line of treatment, and are an alternative to standard chemotherapy in patients with activating mutations of the EGFR gene. The method of qualification for EGFR TKI monotherapy in second- or third-line treatment in NSCLC is still debatable and unclear [10-16].

In the case of the wild type form of the EGFR gene, even if the receptor is overexpressed, EGFR TKI have not shown strong antitumor activity (none or a few percent of objective response) mainly for two reasons: lack of changes in the structure of the ATP-binding pocket of tyrosine kinase domain and the small role of the normal EGFR form in carcinogenesis of lung cancer. Therefore, investigation of the expression of EGFR on the cell surface (by immunohistochemistry) or amplification of the EGFR gene detected by molecular probes and fluorescence in situ hybridization (FISH) did not find a wider application in qualification for EGFR TKI therapy. In some cases, it is possible to consider the usefulness of the determination of EGFR gene amplification by the FISH method, if examination of the mutation is unfeasible or the outcome is uncertain, bearing in mind the frequent co-existence of EGFR gene mutations (10\% of patients) with an increase in copy number of the gene ( $40 \%$ of patients) in tumour cells [7, 8].

Unfortunately, regardless of the applied line of EGFR TKI treatment, at some point of therapy, even in patients who carry an activating mutation in the EGFR gene and show a response, there is a recurrence of the disease and the patient's life is not prolonged $[17,18]$.

\section{Rare mutations in the EGFR gene and resistance to EGFR TKI in NSCLC}

Some mechanisms associated with resistance to reversible EGFR TKI in NSCLC are already known. The T790M mutation in exon 20 of the EGFR gene was detect- ed for the first time in tumour cells of a patient with progression after a few months of efficacious therapy with gefitinib. That point mutation is caused by a single nucleotide substitution (C->T) at position 2369 (codon 790) in exon 20 of the EGFR gene. The T790M mutation leads to a substitution of threonine to methionine in the catalytic centre of EGFR tyrosine kinase, which is located in the binding pocket of TKI and ATP. Substitution of threonine at codon 790 into larger methionine probably results in blocking of the binding sites of erlotinib and gefitinib aromatic residues with their point of action. In vitro studies demonstrated that the effective concentration of erlotinib in tumour cells harbouring both the L858R and T790M mutations has to be 300 times higher to induce apoptosis than in cells only with an activating mutation in codon 858 [19, 20].

Presence of a mutation in exon 20 of the EGFR gene is a potential prognostic and predictive marker of EGFR TKI therapy and may play an important role in the planning of therapy. Maheswaran et al. examined 27 patients with activating mutations in the EGFR gene. These patients were treated with erlotinib or gefitinib. Activating mutations in EGFR were detected in cells obtained from primary tumour, circulating tumour cells in peripheral blood (consistency of results $-92 \%$ ) and in free circulating DNA (consistency of results $-33 \%$ ). The authors also investigated presence of the T790M mutation in similar samples, and they detected cells harbouring that mutation in 10 patients ( $n=26$; 38.5\%). Median survival in patients with the T790M mutation treated with reversible EGFR TKI was 7.7 months against 16.5 months in patients without that mutation $(p<0.001)$. Mutations were detected using SARMS real-time PCR technique. That investigation has also shown that mutation in exon 20 of the EGFR gene leads to acquired resistance to treatment in patients not only with the wild type EGFR gene, but also in patients with confirmed clinical sensitivity to EGFR tyrosine kinase inhibitors [21].

Presence of T790M substitution may be an indication for treatment with irreversible EGFR TKI, such as neratinib, keratinib and especially afatinib, which is currently in the third phase of clinical trials. The LUX-Lung 1 study has shown that afatinib induces an objective response and prolongation of progression-free survival (PFS) in NSCLC patients who have been previously treated with EGFR TKI for at least 12 weeks. The rationale for this type of study is the fact that afatinib has a different molecular structure when compared to gefitinib and erlotinib. This structure causes that afatinib forms a covalent bond with cysteine at codon 733 of the EGFR tyrosine kinase domain. Therefore, inhibition of afatinib is only possible due to synthesis of new receptor proteins. Inhibition of phosphorylation by EGFR tyrosine kinase in tumour cells after afatinib exposure remains active for 48 hours (after gefitinib only 8 hours), and phosphorylation activity of the kinase after that time ranges from 50 to $70 \%$ of the primary activity. In vitro studies and some case reports have shown that afatinib is effective (cell apoptosis and disease stabilisation) in cells harbouring the T790M mutation in NSCLC patients [19, 22-26]. 
The mechanism of mutation appearance in NSCLC recurrence in EGFR TKI treated patients should be thoroughly examined. A resistant cell subpopulation harbouring T790M can emerge in the tumour probably as a result of massive apoptosis of tumour cells harbouring an activating mutation associated with response to therapy. Earlier investigations by Pao et al. did not confirm that observation. The authors examined presence of mutations in exons 18-24 of the EGFR gene in patients treated with EGFR TKI in order to determine if additional mutations in the EGFR gene are associated with progression of the disease. In patients with mutations identified in exon 19 or 21 additional mutations were not reported. Samples obtained from patients' tumour cells before treatment were re-examined to exclude the possibility of missing changes in exon 20 of EGFR. However, no evidence for the presence of the sought mutations was found. Mutations associated with resistance to EGFR TKI were observed in three tumor recurrences that emerged after treatment. It was the T790M mutation coexisting with deletion in exon 19 or L858R substitution in the EGFR gene [20].

Results of the investigations suggest that the resistance to treatment is acquired during therapy with EGFR TKI, and mutations may form de novo. It is worth mentioning that tumour cell clones harbouring T790M substitution may be rare, and the mutation may not be detected (due to the small number of cells with the T790M mutation in the whole sample). It is observed especially when the DNA sequencing technique is employed, which is characterised by relatively low sensitivity and requires the presence of at least $50 \%$ of mutated tumour cells in the examined sample [27].

Currently it is believed that T790M substitution occurs in over $50 \%$ of cases of acquired resistance to reversible EGFR TKI in NSCLC patients and in approximately $5 \%$ of patients before EGFR TKI treatment [15]. Before the therapy with EGFR TKI, the T790M mutation emerges along with activating mutations of the EGFR gene, and secondarily it coexists with the primary activating mutation in EGFR, especially L858R [17, 28-31].

With time, it was found that other mutations in exon 20 and 21 of EGFR (insertion-deletion, substitutions: D761Y, T854A, etc.) may be associated with the mechanism of acquired resistance to EGFR TKI. These mutations account for less than $5 \%$ of all known mutations in the EGFR gene. They are detected very rarely, so reports about their presence should be made carefully, and EGFR TKI treatment outcomes in patients harbouring these mutations should be confirmed on a larger group of patients. Furthermore, it is necessary to understand the mechanism of resistance caused by these mutations [17].

D761Y and T854A mutations in the EGFR gene were detected for the first time in patients with activating L858R mutation. Probably substitution of aspartic acid with tyrosine at position 761 is responsible for acquisition of resistance to TKI treatment of tumour cells with an activating mutation which are sensitive to the treatment (this theory was confirmed in tumour cell lines in vitro). However, this resistance to EGFR TKI has been many times lower than the resistance in cells harbouring both the L858R and T790M mutations. In case of occurrence of both L858R and T790M mutations, the concentration of erlotinib should be three times higher in order to achieve a therapeutic effect. Analysis of the EGFR structure in patients harbouring the T854A mutation suggests the presence of an additional side chain, localised near the EGFR TKI binding site [19]. The role of rare mutations in exon 20 such as S768I and V769L in development of acquired resistance to EGFR TKI is still unknown. These substitutions were reported for the first time in NSCLC patients with progression, bearing activating mutations, after gefitinib therapy. In vitro cultures of cell lines harbouring the S768I substitution showed slightly more resistance to EGFR TKI than cells with wild type EGFR. In these cells permanent hyperphosphorylation of tyrosine at codon 1045 of the tyrosine kinase domain has been observed [17, 32-34].

Insertion-deletion mutations in exon 20 of EGFR are also associated with acquired resistance to EGFR TKI. These mutations, similarly to activating mutations in the EGFR gene, are more often detectable in Asians, non-smokers, women and in patients with adenocarcinoma. Most of the pre-clinical investigations allowed observation of their impact on resistance to therapeutic doses of gefitinib or erlotinib, and some of the irreversible EGFR TKI, e.g. neratinib. However, in the most common mutations such as N771GY and A767-V769 duplication, even though lack of sensitivity to erlotinib has been reported, low sensitivity to irreversible EGFR TKI such as afatinib and PF-00299804 was observed. Crystallographic analysis of epidermal growth factor receptor structure has shown that both mutations have an influence on the spatial structure of tyrosine kinase C-helix, which plays an important role in phosphorylation activity of that enzyme. Other insertions in exon 20 of the EGFR gene such as V738InsKIPVAI, M766InsASV, D770InsNPG and D770InsNPH seem to be associated with that process, but their role in development of resistance to reversible EGFR TKI is still unknown $[35,36]$.

\section{The importance of mutations in the $K$-Ras gene in the planning of EGFR TKI therapy}

Many studies have demonstrated that the presence of mutations at codons 12,13 or 61 in the $K$-Ras gene is not a predictive factor in treatment with EGFR TKI. These observations stem from the fact that mutations in the $K$-Ras gene almost never coexist with mutations in the EGFR gene. Lack of mutations in EGFR determines the inefficiency of EGFR TKI used in therapeutic doses, which means that the occurrence of mutations in the $K$-Ras gene is no longer relevant. Therefore there were no objective responses to treatment with EGFR TKI among carriers of $K$-Ras mutations, which was initially considered as an independent, adverse predictive factor in EGFR TKI treatment. In fact, in vitro studies have shown that the destruction of tumour cells with the wild-type EGFR and mutant $K$-Ras gene is only possible with very high, non-therapeutic doses of EGFR TKI [37-39]. However, in some individual case reports in which coexistence of both EGFR and K-Ras gene mutations have been observed, the efficacy of EGFR TKI was 
not entirely confirmed [40]. The situation is presented in a different manner when monoclonal anti-EGFR antibodies, such as cetuximab, are used in the treatment of colorectal cancer, head and neck cancer, and perhaps soon in NSCLC with strong overexpression of EGFR. Mutations in the $K$-Ras gene are a negative predictor here, and are routinely determined in qualification of patients for therapy. However, in the case of therapy with EGFR TKI the only test eligible for treatment is investigation of EGFR gene mutations [37, 41, 42].

Activation of an alternative signalling pathway from stimulated EGFR protein leads to activation of RAS protein and subsequently kinases associated with RAF/MAPK/ERG proteins and transcription factors such as MYC, FOS and JUN. Mutations which are present in the $K$-Ras gene lead to constant activation of Ras protein, so the tumour cells become independent of the signals from the EGFR, which explains the mechanism of resistance conferred by the EGFR TKI and monoclonal anti-EGFR antibodies. Mutations in the K-Ras gene occur with a frequency of 15-20\% in Caucasian NSCLC patients. Substitutions in codons 12 (G12V, G12C, G12A, G12R, G12D, or G12S) and 13 (G13D) in the K-Ras gene are much more common than in codon 61 and they are mostly observed in smokers with adenocarcinoma (about 30\% of patients with this type of cancer) [37, 38].

\section{Activation of EGFR-independent signalling pathways in cancer cells}

EGFR is not the only receptor on the cell surface responsible for initiation of signal transduction for cell activation and proliferation through Pi3K/AKT and RAS/RAF/MAPK/ERG pathways. Insulin-like growth factor 1 receptor (IGF-1R) and the c-MET receptor for hepatocyte growth factor (HGF) also play an important role in this signal transduction. Overexpression of these receptors and amplification of the genes encoding them often occur in NSCLC cells [43].

Overexpression of C-MET is associated with generation of an additional excitation signal (without EGFR) in protein kinase of AKT, which allows tumour cells to survive and proliferate and induces the formation of metalloproteinases responsible for invasion and metastasis. This mechanism is observed in 3-7\% of patients who were not treated with EGFR $\mathrm{TKI}$ and may explain the initial resistance to this kind of treatment. However, approximately $20 \%$ of patients with EGFR activating mutations develop $M E T$ gene amplification as a result of the creation of acquired resistance to EGFR TKI. Moreover, many patients are characterised by high levels of HGF in tumour tissue. Blocking of c-MET can restore the sensitivity of cancer cells to the EGFR TKI. Unfortunately, more than half of patients with MET gene amplification also presented the T790M mutation in the EGFR gene. Despite the lack of unequivocal efficacy, simultaneous inhibition of c-MET and EGFR function seems to be an attractive alternative therapeutic option in resistance to reversible EGFR TKI. There are ongoing second and third phase trials of the C-MET inhibitors tivantinib and PF-2341066, used together with erlotinib in patients previously untreated with EGFR TKI or in case of progression after successful monotherapy with erlotinib [17, 43-48].
Similarly to the c-MET receptor, the IGF-1R also leads to activation of the AKT pathway without EGFR in NSCLC cells. Development of effective small-molecule inhibitors of IGF$1 R$ encountered difficulties due to the similarity in the tyrosine kinase domain of insulin and IGF-1 receptors. Attempts to use such drugs (e.g. linsitinib) had ended with serious side effects in the form of disorders in carbohydrate and lipid metabolism. Monoclonal anti-IGF-1R antibodies, such as figitumumab, proved to be more selective for the IGF-1R, but were also not devoid of side effects. As a result, attempts of the combined administration of figitumumab and erlotinib were discontinued in 2010 [49-51].

\section{Mutations in the HER2 gene}

Mutations or amplifications of the HER2 gene, whose product as well as EGFR (HER1) belongs to the family of HER receptors, plays an important role in the development of human tumours, such as breast, ovarian and stomach cancers. HER2 compound is a preferential partner in the process of dimerisation for EGFR. Reactions between these receptors may play an important role in pathogenesis of NSCLC. In the case of increased expression of HER2 protein and amplification of its gene (about 20-30\% of patients with NSCLC, mainly patients with adenocarcinoma subtype) the dimerisation between HER2 and EGFR occurs more easily and the ability to increase proliferation of tumour cells is higher. It is also connected with worse prognosis and more aggressive course of the NSCLC [52].

Mutations in the tyrosine kinase domain of HER2 are reported in lung cancer as very rare (about $2-4 \%$, more often in adenocarcinoma, non-smokers and women). They are independent of overexpression of this receptor. They occur as insertiondeletion changes, which change the reading frame in eight codons in exon 20 of the HER2 gene. The most common disorders are insertion-deletion of 12 base pairs: A775VMA (66\% of all discovered mutations in the HER2 gene) and M774AYVM. There are also very rare mutations such as L755S and G776X substitutions and ins776V. These mutations are analogous to a mutation in exon 20 of the EGFR gene and cause changes in the tyrosine kinase domain of the HER2 receptor. On the basis of this similarity, it is assumed that a mutation in the HER2 gene is caused by similar factors as in the EGFR gene and causes similar effects. Narrowing of the ATP binding gap leads to an increase in tyrosine kinase activity and excessive phosphorylation of signalling proteins and subsequent secondary resistance to the reversible EGFR TKI $[53,54]$.

Although the main mechanism of the decreased response to reversible EGFR TKI is caused by mutations in exon 20 (T790M) of the EGFR gene, equally strong resistance to reversible EGFR TKI may be caused by insertion-deletion changes in exon 20 of the HER2 gene. However, studies of this phenomenon have been carried out only in cell cultures [53-55].

EGFR TKI, which are effective for the T790M mutation of the EGFR gene, may also prove to be effective in patients with mutations in exon 20 of the HER2 gene, but only if they have the ability to block the function of the tyrosine kinase as- 
sociated with both EGFR and HER2. A double reversible inhibitor of EGFR and HER2 receptors called lapatinib has not shown efficacy in this case. However, irreversible inhibitors, such as afatinib and neratinib, which additionally have a likely inhibition effect on the HER4 tyrosine kinase, may be effective in the case of mutations in both genes. Mutations in exon 20 of the HER2 gene do not eliminate the possibility of afatinib binding to the tyrosine kinase domain, as the drug is covalently associated with cysteine at codon 805 of this domain. In in vitro cultures afatinib is characterised by about 10 -fold greater ability to inhibit phosphorylation than gefitinib in case of mutations in exon 20 of EGFR or HER2 genes. Also several cases have been reported where afatinib was effective in NSCLC patients with mutations in exon 20 of the HER2 gene. There are also premises about the effectiveness of combined use of afatinib and mTOR inhibitors (mammalian target of rapamycin) if HER2 mutation is present. Signalling mTOR protein is activated by the Pi3K/AKT cascade and provides the signal which regulates both mRNA translation and cell growth. Its inhibition by such agents as temsirolimus, everolimus or deforolimus can increase the potency of dual inhibitors of EGFR and HER2 and overcome the resistance to their use $[25,53-55]$.

\section{Directions for further studies}

The greatest hope for an extension of progression-free survival in patients treated with reversible EGFR TKI, in whom resistance has developed, is provided by test results on the effectiveness of irreversible EGFR TKI. In vitro studies have shown that these drugs exhibit activity against cells with the wild-type or mutated (with activating mutation) form of the EGFR gene, as well as against cells which are resistant to them because of the presence of the T790M mutation in the EGFR gene and insertion- deletion changes in the HER2 gene. However, in the case of wild-type EGFR and mutations which cause resistance to drugs, the concentration of agents required to effectively induce apoptosis in tumour cells may exceed the tolerated dose level in therapy. Currently, it is known that afatinib, which is in the most advanced trials, is effective in some patients who have benefited from pre-treatment with reversible EGFR TKI. Therefore, the expansion of molecular tests with studies concerning mechanisms of resistance to reversible EGFR TKI is necessary. Determination of presence of resistance mutations that cause insensitivity of cancer cells to reversible EGFR TKI, but which does not rule out the effectiveness of irreversible EGFR TKI, may be considered as a qualifying factor for changing the type of therapy [55-57].

There are also investigations on the effectiveness of other irreversible EGFR TKI such as PF-00299802, EKB-569, canertinib (Cl-1033) and neratinib (HKI-272). All of these agents are required in relatively high doses in order to overcome the resistance to EGFR TKI in cancer cells. However, an additional advantage of the irreversible EGFR TKI is their ability to suppress other receptors of the HER group (pan-ErbB inhibitors), which are necessary for heterodimerisation with EGFR. Use of irreversible EGFR TKI and monoclonal anti-EGFR (cetuximab) antibodies or concomitant administration of EGFR and
c-MET inhibitors may present prospects for the future. The results of clinical trials of new generation drugs are promising and their usefulness in molecularly targeted therapies will probably be proven in the near future [55-57].

The authors declare no conflict of interest.

\section{References}

1. Jemal A, Bray F, Centem MM, Ferlay J, Ward E, Forman D. Global cancer statistics. CA Cancer J Clin 2011; 61: 69-90.

2. Jassem J, Biernat W, Drosik K, et al. Uaktualnione zalecenia dotyczace systemowego leczenia niedrobnokomórkowego raka płuca i złośliwego międzybłoniaka opłucnej. Pneumonol Alergol Pol 2010; 78: 418-31.

3. NSCLC Meta-Analysis Collaborative Group. Chemotherapy in addition to supportive care improves survival in advanced non-smallcell lung cancer. A systemic review and meta-analysis of individual patient data from 16 randomized controlled trials. J Clin Oncol 2008; 26: 4617-25.

4. Yun CH, Boggon TJ, Li Y, et al. Structures of lung cancer-derived EGFR mutants and inhibitor complexes: Mechanism of activation and insights into differential inhibitor sensitivity. Cancer Cell 2007; 11: 217-27.

5. Lynch TJ, Bell DW, Sordella R, et al. Activating mutations in the epidermal growth factor receptor underlying responsiveness of nonsmall-cell lung cancer to gefitinib. N Engl J Med 2004; 350: 2129-39.

6. Shigematsu H, Lin L, Takahashi T, et al. Clinical and biological features associated with epidermal growth factor receptor gene mutations in lung cancer. J Natl Cancer Inst 2005; 97: 339-46.

7. Paez JG, Janne PA, Lee JC, et al. EGRF mutations in lung cancer: correlation with clinical response to gefitinib therapy. Science 2004; 304: 1497-500.

8. Pao W, Miller V, Zakowski M, et al. EGF receptor gene mutations are common in lung cancers from "never smokers" and are associated with sensitivity of tumors to gefitinib and erlotinib. Proc Natl Acad Sci U S A 2004; 101: 13306-11.

9. Salgia R, Hensing T, Campbell N, Salama AK, Maitland M, Hoffman P, Villaflor V, Vokes EE. Personalized treatment of lung cancer. Semin Oncol 2011; 38: 274-83.

10. Shepherd FA, Rodrigues-Pereira J, Ciuleanu T, et al. National Cancer Institute of Canada Clinical Trials Group. Erlotinib in previously treated nonsmall-cell lung cancer. N Engl J Med 2005; 353: 123-32.

11. Douillard JY, Shepherd FA, Hirsh V, et al. Molecular predictors of outcome with gefitinib and docetaxel in previously treated non-smallcell lung cancer: data from the randomized phase III INTEREST trial. J Clin Oncol 2010; 28: 744-52.

12. Mok TS Wu YL, Thongprasert S, et al. Gefitinib or carboplatinpaclitaxel in pulmonary adenocarcinoma. N Engl J Med 2010; 361: 947-57.

13. Mitsudomi T, Morita S, Yatabe Y, et al. Gefitinib versus cisplatin plus docetaxel in patients with non-small-cell lung cancer harbouring mutations of the epidermal growth factor receptor (WJTOG3405): an open label, randomized phase 3 trial. Lancet Oncol 2010; 11: 121-8.

14. Zhou C, Wu YL, Chen G, et al. Erlotinib versus chemotherapy as firstline treatment for patients with advanced EGFR mutation-positive nonsmall-cell lung cancer (OPTIMAL, CTONG-0802): a multicentre, openlabel, randomised, phase 3 study. Lancet Oncol 2011; 12: 735-42.

15. Rossel R, Gervias V, Vergnenegre A. Erlotinib vs chemotherapy (CT) in advanced non-small-cell lung cancer (NSCLC) patients ( $p$ ) with epidermal growth factor receptor (EGFR) activating mutations: interim results of the European Tarceva vs Chemotherapy (EURTAC) phase III randomized trial. J Clin Oncol 2011; 29 (suppl.): abstr. 7503.

16. Paz-Ares L, Soulieres D, Melezinek I, et al. Clinical outcomes in nonsmall-cell lung cancer patients with EGFR mutations: pooled analysis. Cell Mol Med 2010; 14: 51-69.

17. Kosaka T, Yamaki E, Mogi A, Kuwano H. Mechanisms of resistance to EGFR TKIs and development of a new generation of drugs in nonsmall-cell lung cancer. J Biomed Biotechnol 2011; 2011: 165214. 
18. Daub H, Specht K, Ullrich A. Strategies to overcome resistance to targeted protein kinase inhibitors. Nat Rev Cancer 2004; 3: 1001-10.

19. Avizienyte E, Ward RA, Garner AP. Comparison of the EGFR resistance mutation profiles generated by EGFR targeted tyrosine kinase inhibitors and the impact of drug combinations. Biochem J 2008; 415: 197-206.

20. Pao W, Miller VA, Politi KA, Riely GJ, Riely GJ, Somwar R, Zakowski MF, Kris MG, Varmus H. Acquired resistance of lung adenocarcinomas to gefitinib or erlotinib is associated with a second mutation in the EGFR kinase domain. PLoS Medicine 2005; 23: 225-35.

21. Maheswaran S, Sequist LV, Nagrath S, et al. Detection of mutations in EGFR in circulating lung-cancer cells. N Engl J Med 2008; 359: 366-77.

22. Doebele RC, Oton AB, Peled N, et al. New strategies to overcome limitations of reversible EGFR tyrosine kinase inhibitor therapy in non-small cell lung cancer. Lung Cancer 2010; 69: 1-12.

23. Kwak EL, Sordella R, Bell DW, et al. Irreversible inhibitors of the EGF receptor may circumvent acquired resistance to gefitinib. Proc Nat Acad Sci U S A 2005; 102: 7665-70.

24. Hirsh V. Afatinib (BIBW 2992) development in non-small-cell lung cancer. Future Oncol 2011; 7: 817-25.

25. Li D, Ambrogio L, Shimamura T. BIBW2992, an irreversible EGFR/HER2 inhibitor highly effective in preclinical lung cancer models. Oncogene 2009; 27: 4702-11.

26. Zhou W, Ercan D, Chen L, et al. Novel mutant-selective EGFR kinase inhibitors against EGFR T790M. Nature 2009; 462: 1070-4.

27. Ellison G, Donald E, McWalter G, et al. A comparison of ARMS and DNA sequencing for mutation analysis in clinical biopsy samples. J Exp Clin Cancer Res 2010; 29: 132-9.

28. Inukai M, Toyooka S, Ito S, et al. Presence of epidermal growth fac tor receptor gene $T 790 \mathrm{M}$ mutation as a minor clone in non-smallcell lung cancer. Cancer Res 2006; 66: 7854-8.

29. Wu JY, Wu SG, Yang CH, et al. Lung cancer with epidermal growth factor receptor eon 20 mutations is associated with poor gefitinib treatment response. Clin Cancer Res 2008; 14: 4877-82.

30. Yun $\mathrm{CH}$, Mengwasser KE, Toms AV, et al. The T790M mutation in EGFR kinase causes drug resistance by increasing the affinity for ATP. Proc Natl Acad Sci U S A 2008; 105: 2070-5.

31. Ma C, Wei S, Song Y. T790M and acquired resistance of EGFR TKI: a literature review of clinical reports. J Thorac Dis 2011; 3: 10-8.

32. Bean J, Riely GJ, Balak MN, et al. Acquired resistance to epidermal growth factor receptor kinase inhibitors associated with a nove T854A mutation in a patient with EGFR-mutant lung adenocarcinoma. Clin Cancer Res 2008; 14: 7519-25.

33. Asahina H, Yamazaki K, Kinoshita I, Yokouchi H, Dosaka-Akita H, Nishimura M. Non-responsiveness to gefitinib in a patient with lung adenocarcinoma having rare EGFR mutations S768I and V769L Lung Cancer 2006; 54: 419-22

34. Balak MN, Gong Y, Riely GJ, et al. Novel D761Y and common secondary T790M mutations in epidermal growth factor receptor-mutant lung adenocarcinoma with acquired resistance to kinase inhibitors. Clin Cancer Res 2006; 12: 6494-501.

35. asuda H, Kobayashi S, Costa DB. EGFR exon 20 insertion mutations in non-small-cell lung cancer: preclinical data and clinical implications. Lancet Oncol 2012; 13: e23-31.

36. Harada T, Lopez-Chavez A, Xi L, Raffeld M, Wang Y, Giaccone G. Characterization of epidermal growth factor receptor mutations in non-small-cell lung cancer patients of African-American ancestry. Oncogene 2011; 30: 1744-52.

37. Schneider C, Schott-von Römer K, Gütz S i wsp. Biomarker analysis from TRUST, a trial of erlotinib in non-small cell lung cancer (NSCLC) J Clin Oncol 2007; 25 (suppl.): abstr. 7674

38. Linardou H, Dahabreh IJ, Kanaloupiti D, Siannis F, Bafaloukos D, Kosmidis P, Papadimitriou CA, Murray S. Assessment of somatic k-RAS mutations as a mechanism associated with resistance to EGFR-targeted agents: a systematic review and meta-analysis of studies in advanced non-small-cell lung cancer and metastatic colorectal cancer. Lancet Oncol 2008; 9: 962-72.

39. Pao W, Wang TY, Riely GJ, et al. KRAS mutations and primary resis tance of lung adenocarcinomas to gefitinib or erlotinib. PLoS Med 2005; 2: 57-61.

40. Gumerlock PH, Holland WS, Chen H, et al. Mutational analysis of K-RAS and EGFR implicates K-RAS as a resistance marker in the Southwest Oncology Group (SWOG) trial S0126 of bronchioalveo- lar carcinoma (BAC) patients (pts) treated with gefitinib. J Clin Oncol 2005; 23 (suppl.): abstr. 7008.

41. Zhang X, Chang A. Molecular predictors of EGFR-TKI sensitivity in advanced non-small cell lung cancer. Int J Med Sci 2008; 5: 209-17.

42. Toschi L, Cappuzzo F. Understanding the new genetics of responsiveness to epidermal growth factor receptor tyrosine kinase inhibitors. Oncologist 2007; 12: 211-20.

43. Mitsudomi T, Yatabe Y. Mutations of the epidermal growth facto receptor gene and related genes as determinants of epidermal growth factor receptor tyrosine kinase inhibitors sensitivity in lung cancer. Cancer Sci 2007; 98: 1817-24.

44. Kubo T, Yamamoto H, Lockwood WW, et al. MET gene amplification or EGFR mutation activate MET in lung cancers untreated with EGFR tyrosine kinase inhibitors. Int J Cancer 2009; 124: 1778-84.

45. Turke AB, Zejnullahu K, Wu YL, et al. Preexistence and clonal selection of MET amplification in EGFR mutant NSCLC. Cancer Cell 2010; 17: 77-88.

46. Bean J, Brennan C, Shih JY, et al. MET amplification occurs with or without T790M mutations in EGFR mutant lung tumors with acquired resistance to gefitinib or erlotinib. Proc Natl Acad Sci U S A 2007; 104: 20932-7.

47. Yano S, Wang W, Li QI, et al. Hepatocyte growth factor induces gefitinib resistance of lung adenocarcinoma with epidermal growth factor receptor-activating mutations. Cancer Res 2008; 68: 9479-87.

48. Sequist LV, von Pawel J, Garmey EG, et al. Randomized phase II study of erlotinib plus tivantinib versus erlotinib plus placebo in previously treated non-small-cell lung cancer. J Clin Oncol 2011; 29: 3307-15.

49. Gridelli C, Rossi A, Bareschino MA, Schettino C, Sacco PC, Maione P. The potential role of insulin-like growth factor receptor inhibitors in the treatment of advanced non-small cell lung cancer. Expert Opin Investig Drugs 2010; 19: 631-9.

50. Guix M, Faber AC, Wang SE i wsp. Acquired resistance to EGFR tyrosine kinase inhibitors in cancer cells is mediated by loss of IGF-binding proteins. J Clin Invest 2008; 118: 2609-19.

51. Gridelli C, Rossi A, Bareschino MA, Schettino C, Sacco PC, Maione P. The potential role of insulin-like growth factor receptor inhibitors in the treatment of advanced non-small cell lung cancer. Expert Opin Investig Drugs 2010; 19: 631-9.

52. Hirsch FR, Varella-Garcia M, Cappuzzo F. Predictive value of EGFR and HER2 overexpression in advanced non-small-cell lung cancer. Oncogene 2009; 28 (suppl.): 32-7.

53. Shigematsu H, Takahashi T, Nomura M, et al. Somatic Mutations of the HER2 Kinase Domain in Lung Adenocarcinomas. Cancer Res 2005; 65: 1642-6.

54. Perera SA, Danan L, Shimamura T, et al. HER2YVMA drives rapid development of adenosquamous lung tumor in mice that are sensitive to BIBW2992 and rapamycin combination therapy. PNAS 2009; 2: 474-9.

55. Hirsh V. Afatinib (BIBW 2992) development in non-small-cell lung cancer. Future Oncol 2011; 7: 817-25.

56. Giaccone G, Wang Y. Strategies for overcoming resistance to EGFR family tyrosine kinase inhibitors. Cancer Treat Rev 2011; 37: 456-64.

57. Bonanno L, Jirillo A, Favaretto A. Mechanisms of acquired resistance to epidermal growth factor receptor tyrosine kinase inhibitors and new therapeutic perspectives in non small cell lung cancer. Curr Drug Targets 2011; 12: 922-33.

\section{Address for correspondence}

Prof. Paweł Krawczyk MD, PhD

Department of Pneumonology, Oncology and Allergology

Medical University of Lublin

Jaczewskiego 8

20-954 Lublin

tel. +48817244293

e-mail: krapa@poczta.onet.pl

Submitted: $\quad 27.11 .2011$

Accepted: $\quad 2.04 .2012$ 